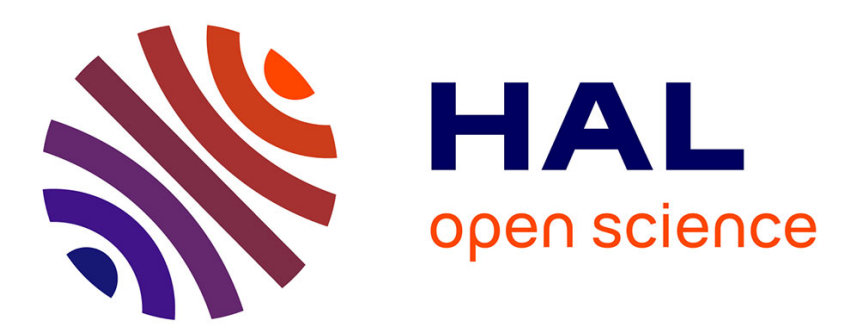

\title{
A Cahn-Hilliard system with a fidelity term for color image inpainting
}

Laurence Cherfils, Hussein Fakih, Alain Miranville

\section{To cite this version:}

Laurence Cherfils, Hussein Fakih, Alain Miranville. A Cahn-Hilliard system with a fidelity term for color image inpainting. 2015. hal-01138569

\section{HAL Id: hal-01138569 \\ https://hal.science/hal-01138569}

Preprint submitted on 2 Apr 2015

HAL is a multi-disciplinary open access archive for the deposit and dissemination of scientific research documents, whether they are published or not. The documents may come from teaching and research institutions in France or abroad, or from public or private research centers.
L'archive ouverte pluridisciplinaire HAL, est destinée au dépôt et à la diffusion de documents scientifiques de niveau recherche, publiés ou non, émanant des établissements d'enseignement et de recherche français ou étrangers, des laboratoires publics ou privés. 


\title{
A Cahn-Hilliard system with a fidelity term for color image inpainting
}

\author{
Laurence Cherfils*|, Hussein Fakih ${ }^{\dagger} \|$, and Alain Miranville ${ }^{\ddagger} \|$ \\ Université de la Rochelle \\ Laboratoire Mathématiques, Image et Applications \\ Avenue Michel Crépeau \\ F-17042 La Rochelle Cedex, France \\ II Université de Poitiers \\ Laboratoire de Mathématiques et Applications \\ UMR CNRS 7348 - SP2MI \\ Boulevard Marie et Pierre Curie - Téléport 2 \\ F-86962 Chasseneuil Futuroscope Cedex, France
}

\begin{abstract}
In this paper, we propose a model for multi-color image inpainting composed of $n$ colors. In particular, as in the binary model, i.e., the Bertozzi-EsedogluGillette-Cahn-Hilliard equation [4], we add a fidelity term to the corresponding Cahn-Hilliard system. We are interested in the study of the asymptotic behavior, in terms of finite-dimensional attractors, of the dynamical system associated with the problem. The main difficulty here is that we no longer have the conservation of mass, i.e., of the spatial average of the order parameter $c$, as in the CahnHilliard system. Instead, we prove that the spatial average of $c$ is dissipative. We finally give numerical simulations which confirm and extend previous ones on the efficiency of the binary model.
\end{abstract}

Keywords: Cahn-Hilliard system, fidelity term, color image inpainting, wellposedness, exponential attractor, simulations.

AMS Subject Classification: 35B40; 35K55; 68M07; 80A23

\section{Introduction}

Image inpainting involves filling in part of an image or video from the surrounding area. It is essentially some kind of interpolation. Its applications include restoration of old paintings by museum artists [28], removing scratches from old photographs [8], altering scenes in photographs [37], and restoration of motion pictures [38].

The authors in [3] introduced image inpainting for digital image processing. Their model is based on nonlinear PDE's. A large number of PDE's and variational approaches

\footnotetext{
${ }^{*}$ E-mail address: Laurence.Cherfils@univ-lr.fr

${ }^{\dagger}$ E-mail address: Hussein.Fakih@math.univ-poitiers.fr

${ }^{\ddagger}$ E-mail address: Alain.Miranville@math.univ-poitiers.fr
} 
have been considered in view of such applications, such as the total variation model $[11,12,13,21,43,44,45]$, the Euler elastica model [10, 14, 40], the active contour model based on the Mumford and Shah segmentation [47], the inpainting scheme based on the Mumford-Shah-Euler image model [29], inpainting with the Navier-Stokes equations [2], or wavelet-based inpainting [14, 20].

The authors of [4] introduced the following Cahn-Hilliard model for binary image inpainting:

$$
\frac{\partial c}{\partial t}=-\Delta\left(\varepsilon \Delta c-\frac{1}{\varepsilon} f(c)\right)+\lambda(x)(h-c),
$$

where

$$
\lambda(x)=\left\{\begin{array}{lll}
0 & \text { if } & x \in D, \\
\lambda_{0} & \text { if } & x \in \Omega \backslash D,
\end{array}\right.
$$

$h(x)$ is a given binary image, and $D \Subset \Omega$ is the inpainting domain. Here, $c$ satisfies the Neumann boundary conditions

$$
\frac{\partial c}{\partial \nu}=\frac{\partial \Delta c}{\partial \nu}=0, \quad \text { on } \partial \Omega,
$$

and $F$ is the antiderivative of $f$ such that

$$
F(s)=s^{2}(s-1)^{2} .
$$

Equation (1.1) is identical to the standard Cahn-Hilliard equation [1, 42], except for the second term on the right-hand side. This term is added to keep the solution constructed close to the given image $h(x)$ in the complement of the inpainting domain, where image information is available.

Well-posedness results for (1.1) have been obtained in [4] (see also [9] for the study of the stationary problem).

Furthermore, the asymptotic behavior, in terms of finite-dimensional attractors, of (1.1) has been studied in [15]. More precisely, such sets provide information on all the possible dynamics of the system and are expected to have a rich geometric structure; they contain in particular all steady states and heteroclinic orbits. Moreover, the finitedimensionality means, roughly speaking, that, even though the initial phase space is infinite-dimensional, the reduced dynamics can be characterized by a finite number of parameters. We refer the reader to, e.g., $[24,41,46]$ for discussions on this subject.

Finally, the existence of local (in time) solutions to (1.1) with logarithmic nonlinear terms has been studied in [16] (note indeed that the original Cahn-Hilliard equation was actually proposed with thermodynamically relevant logarithmic nonlinear terms which follow from a mean-field model; regular (and, in particular, cubic) nonlinear terms are approximations of such logarithmic nonlinear terms). In that case, we can obtain better results than those obtained with polynomial nonlinear terms in [15], as far as the convergence time is concerned, in particular. We also note that double obstacle nonlinear terms give better results than those obtained with polynomial nonlinear terms, see [5].

While the articles mentioned above only deal with binary images (i.e., black and white; see however [9] for grayvalue images), it is natural and important to also address color images. In particular, one idea in this direction is to extend the approach of [4] to multi-component Cahn-Hilliard systems, each phase corresponding to a given color. Multi-component Cahn-Hilliard systems have been proposed and studied in, 
e.g., $[19,27,30,34,39]$. Our main aim in this article is to propose such a model for color image inpainting, based on a fidelity term as in [4].

As far as the mathematical treatment of the problem is concerned, we have to face two essential difficulties. The first one, which we already encountered in the binary model in [15], is that, due to the presence of the fidelity term, we no longer have the conservation of mass, i.e., of the spatial average of the order parameter, contrary to the original Cahn-Hilliard system. This is overcome by deriving a global (in time) and dissipative (i.e., independent of time and bounded sets of initial data, at least for large times) estimate on the order parameter; in particular, a key step is to obtain such an estimate on the spatial average of the order parameter. We can note that this estimate is a crucial one, already in order to prove the existence of a solution. The second difficulty is that the order parameter has to satisfy some constraint, namely, the sum of the phases must be equal to one. As a consequence, we have to be careful in the choice of the functional spaces and in the (weak) formulation of the problem.

This article is organized as follows. In Section 2, we present the model constructed from the multi-phase Cahn-Hilliard system by adding a fidelity term. Furthermore, we give the assumptions on the nonlinear term. Then, in Section 3, we derive a priori estimates which allow us to prove, in Section 4, the existence and uniqueness of solutions, as well as the existence of the global attractor. In Section 5, we construct finite-dimensional attractors and, in Section 6, we prove that the model is algebraically consistent with the diphasic one. Finally, in Section 7, we give some numerical simulations which confirm that the one step algorithm with threshold proposed in [15] (see also [16]) is efficient, also in the context of multi-color inpainting.

Remark 1.1. After this work was completed, we discovered the recent preprint [6] in which a similar model is considered for grayvalue inpainting. Note however that, there, the mathematical analysis of the problem is not addressed.

\section{Proposed model and setting of the problem}

Let $c=\left(c_{1}, c_{2}, \ldots, c_{n}\right)$ be the phase variable and $\Omega$ be a bounded domain of $\mathbb{R}^{N}(N=$ $2,3)$ with a regular boundary $\Gamma$. We assume that $\sum_{i=1}^{n} c_{i}=1$ and $0 \leq c_{i} \leq 1, i=1, \ldots, n$. We define the hyperplane

$$
S:=\left\{c \in \mathbb{R}^{n} \text { such that } \sum_{i=1}^{n} c_{i}=1\right\} .
$$

We postulate that the free energy can be written as follows:

$$
\mathcal{F}=\int_{\Omega}\left[F(c)+\frac{\varepsilon^{2}}{2} \sum_{i=1}^{n}\left|\nabla c_{i}\right|^{2}\right] d x,
$$

where $F(c)=\frac{1}{n} \sum_{i=1}^{n} c_{i}^{2}\left(1-c_{i}\right)^{2}$. The time evolution of $c$ is governed by the gradient of the energy with respect to the $H^{-1}$-inner product under the additional constraint (2.1). This constraint has to hold everywhere and at every time. In order to ensure this constraint, we use a variable Lagrangian multiplier $\beta(c)$ [34]. The time dependence of $c_{i}$ is given by the following system of Cahn-Hilliard equations describing each phase:

$$
\frac{\partial c_{i}}{\partial t}=\Delta \mu_{i}, \quad i=1, \ldots, n,
$$




$$
\mu_{i}=\frac{\partial F(c)}{\partial c_{i}}-\varepsilon^{2} \Delta c_{i}+\beta(c), \quad i=1, \ldots, n .
$$

To compute $\beta(c)$, we write the equation satisfied by $C=c_{1}+c_{2}+\ldots+c_{n}$ and we want $C=1$ to be solution to this equation,

$$
\frac{\partial C}{\partial t}=\Delta\left(\sum_{i=1}^{n} \frac{\partial F}{\partial c_{i}}-\varepsilon^{2} \Delta C+n \beta(c)\right) .
$$

Therefore, $\beta(c)=-\frac{1}{n} \sum_{i=1}^{n} \frac{\partial F}{\partial c_{i}}$ and we obtain

$$
\begin{gathered}
\frac{\partial c_{i}}{\partial t}=\Delta \mu_{i}, \quad i=1, \ldots, n, \\
\mu_{i}=\frac{\partial F(c)}{\partial c_{i}}-\varepsilon^{2} \Delta c_{i}-\frac{1}{n} \sum_{i=1}^{n} \frac{\partial F}{\partial c_{i}}, \quad i=1, \ldots, n .
\end{gathered}
$$

Finally, we need to add the fidelity term $\lambda(x)\left(h_{i}-c_{i}\right)$ to each equation to keep the solution constructed close to the given image $h_{i}(x)$ as in the Bertozzi-EsedogluGillette-Cahn-Hilliard model for binary image inpainting; more precisely, $h_{i}(x)$ is a part of the original image corresponding to the color representing the phase $c_{i}$. We thus have the following model:

$$
\begin{gathered}
\frac{\partial c_{i}}{\partial t}=\Delta \mu_{i}+\lambda(x)\left(h_{i}-c_{i}\right), \quad i=1, \ldots, n, \\
\mu_{i}=\frac{\partial F(c)}{\partial c_{i}}-\varepsilon^{2} \Delta c_{i}-\frac{1}{n} \sum_{i=1}^{n} \frac{\partial F}{\partial c_{i}}, \quad i=1, \ldots, n,
\end{gathered}
$$

where

$$
\lambda(x)=\left\{\begin{array}{lll}
0 & \text { if } & x \in D, \\
\lambda_{0} & \text { if } & x \in \Omega \backslash D,
\end{array}\right.
$$

$D \Subset \Omega$ is the inpainting domain, and $h=\left(h_{1}, \ldots, h_{n}\right) \in\left(L^{2}(\Omega)\right)^{n}$.

Equations (2.8)-(2.9) are endowed with the Neumann boundary conditions

$$
\frac{\partial c_{i}}{\partial \nu}=\frac{\partial \mu_{i}}{\partial \nu}=0, \quad \text { on } \partial \Omega, i=1, \ldots, n .
$$

We note that the additional fidelity term in (2.8) can be derived from a gradient flow under the $L^{2}$-inner product for the energy

$$
\frac{\lambda_{0}}{2} \sum_{i=1}^{n} \int_{\Omega \backslash D}\left(c_{i}-h_{i}\right)^{2} d x .
$$

Our proposed model (2.8)-(2.9) can thus be thought of as the superposition of a gradient descent with respect to the $H^{-1}$-inner product for (2.2) and a gradient descent with respect to the $L^{2}$-inner product for (2.11). However, it is not the gradient descent, neither in the $H^{-1}$ - nor the $L^{2}$-inner product, for the sum of the energies (2.2) and (2.11). 
We again assume that $\sum_{i=1}^{n} c_{i}=1$, so that the additional fidelity term in (2.8) must also satisfy the constraint (2.1) everywhere and at every time,

$$
\sum_{i=1}^{n} h_{i}=1
$$

hence

$$
\lambda(x) \sum_{i=1}^{n}\left(c_{i}-h_{i}\right)=0, \quad \forall t \geq 0 .
$$

We rewrite (2.8)-(2.10) in the form

$$
\begin{gathered}
\frac{\partial c}{\partial t}=\Delta \mu+\lambda_{0} \chi_{\Omega \backslash D}(x)(h-c), \\
\mu=f(c)-\varepsilon^{2} \Delta c, \\
\frac{\partial c}{\partial \nu}=\frac{\partial \mu}{\partial \nu}=0,
\end{gathered}
$$

where $c=\left(c_{i}\right)_{i}, \mu=\left(\mu_{i}\right)_{i}, h=\left(h_{i}\right)_{i}$, and $f(c)=\left(f_{i}(c)\right)_{i}$,

$$
f_{i}(c)=\frac{2}{n}\left[c_{i}\left(1-c_{i}\right)^{2}-c_{i}^{2}\left(1-c_{i}\right)\right]-\frac{1}{n} \sum_{i=1}^{n} \frac{\partial F}{\partial c_{i}},
$$

$i=1, \ldots, n$.

We define the tangent space of $S$ (defined in (2.1)) as

$$
T S:=\left\{\phi=\left(\phi_{i}\right)_{i} \in \mathbb{R}^{n} \text { such that } \sum_{i=1}^{n} \phi_{i}=0\right\} .
$$

Then, we introduce the projection

$$
\begin{aligned}
\mathbb{P}: \mathbb{R}^{n} & \rightarrow T S \\
\phi & \mapsto \mathbb{P} \phi=\phi-\frac{1}{n}(\phi . e) e,
\end{aligned}
$$

where $e=(1, \ldots, 1)$. Notice that, if $\phi \in S \cap\left(L^{2}(\Omega)\right)^{n}$, then

$$
\mathbb{P} \bar{\phi}=\bar{\phi} .
$$

We denote by $\langle\phi\rangle$ the spatial average of a function $\phi$ in $\left(L^{1}(\Omega)\right)^{n}$,

$$
\langle\phi\rangle:=\frac{1}{\operatorname{Vol}(\Omega)} \int_{\Omega} \phi(x) d x
$$

and set

$$
\bar{c}:=c-\langle c\rangle,
$$

$\bar{c}$ being the mean-free part of $c$.

We further introduce the following spaces:

$$
L=\left(H^{-1}(\Omega)\right)^{n} \cap S, \quad H=\left(L^{2}(\Omega)\right)^{n} \cap S,
$$




$$
\begin{gathered}
V=\left(H^{1}(\Omega)\right)^{n} \cap S, W=\left\{v \in\left(H^{2}(\Omega)\right)^{n} \cap S, \frac{\partial v}{\partial \nu}=0 \text { on } \Gamma\right\}, \\
\dot{H}^{-1}(\Omega)=\left\{\phi \in\left(H^{-1}(\Omega)\right)^{n} \cap T S,\langle\phi, e\rangle_{\left.\left(H^{-1}(\Omega)\right)^{n},\left(H^{1}(\Omega)\right)^{n}=0\right\}},\right. \\
\dot{H}=\left\{\phi \in\left(L^{2}(\Omega)\right)^{n} \cap T S,\langle\phi\rangle=0\right\}, \\
\dot{V}=\left\{\phi \in\left(H^{1}(\Omega)\right)^{n} \cap T S,\langle\phi\rangle=0\right\},
\end{gathered}
$$

and

$$
\dot{W}=\left\{\phi \in\left(H^{2}(\Omega)\right)^{n} \cap T S,\langle\phi\rangle=0\right\} .
$$

We finally introduce the bilinear form

$$
a(u, v)=((\nabla u, \nabla v))
$$

which is continuous on $V$. Here and below, we denote by $\|$.$\| the usual L^{2}-$ norm (with associated scalar product $((.,))$.$) ; in general, \|\cdot\|_{X}$ denotes the norm on the Banach space $X$. We can associate with $a$ the linear operator $A$ in $\dot{V}$ with domain $D(A)=\dot{W}$. For $u \in D(A), A u$ is defined by

$$
((A u, v))=a(u, v), \quad \forall v \in \dot{V},
$$

which amounts to saying that $A u=-\Delta u$. It is easy to show that $A$ is an isomorphism from $\dot{V}$ onto $\dot{V}^{\prime}$ (the dual of $\dot{V}$ ) and from $D(A)$ onto $\dot{H}$. Furthermore, in light of the compact injection of $\left(H^{1}(\Omega)\right)^{n}$ in $\left(L^{2}(\Omega)\right)^{n}$ (and also of $\dot{V}$ in $\dot{H}$ ), it follows that $A^{-1}$ is self-adjoint and compact. There thus exists an orthonormal basis of $\dot{H}$ associated with the eigenvalues $\lambda_{j}, j \geq 1$, of $A$,

$$
\left\{\begin{aligned}
\lambda_{1} & =\inf _{v \in \dot{V} \backslash\{0\}} \frac{\|v\|_{V}^{2}}{\|v\|^{2}}, \\
A N_{j} & =\lambda_{j} N_{j}, \quad N_{j} \in D(A), \quad j \geq 1 \\
0 & <\lambda_{1} \leq \lambda_{2} \ldots
\end{aligned}\right.
$$

The family $\left\{N_{j}\right\}_{j}$ may be assumed to be normalized in the norm of $\dot{H}$, i.e.,

$$
\left(\left(N_{i}, N_{j}\right)\right)=\delta_{i j}
$$

where

$$
\delta_{i j}= \begin{cases}1 & \text { if } i=j, \\ 0 & \text { otherwise. }\end{cases}
$$

We now rewrite problem (2.12)-(2.13) in the form

$$
\begin{gathered}
\frac{\partial c}{\partial t}+A \overline{\mathbb{P} \mu}+\lambda_{0} \chi_{\Omega \backslash D}(x)(c-h)=0, \\
\mathbb{P} \mu=\mathbb{P} f(c)+\varepsilon^{2} A \bar{c} .
\end{gathered}
$$

For simplicity, we set $\varepsilon=1$ and have

$$
\begin{gathered}
\frac{\partial c}{\partial t}+A \overline{\mathbb{P} \mu}+\lambda_{0} \chi_{\Omega \backslash D}(x)(c-h)=0, \\
\mathbb{P} \mu=\mathbb{P} f(c)+A \bar{c} .
\end{gathered}
$$


Integrating (2.18) over $\Omega$, we obtain

$$
\frac{d\langle c\rangle}{d t}=-\lambda_{0}\left\langle\chi_{\Omega \backslash D}(x)(c-h)\right\rangle
$$

We can thus rewrite (2.18)-(2.19) in the form

$$
\begin{gathered}
\frac{\partial \bar{c}}{\partial t}+A \overline{\mathbb{P} \mu}+\lambda_{0} \overline{\chi_{\Omega \backslash D}(x)(c-h)}=0, \\
\mathbb{P} \mu=\mathbb{P} f(c)+A \bar{c} .
\end{gathered}
$$

Finally, we can reformulate the problem as follows:

$$
\begin{gathered}
\frac{\partial A^{-1} \bar{c}}{\partial t}+\overline{\mathbb{P} \mu}+\lambda_{0} A^{-1}\left(\overline{\chi_{\Omega \backslash D}(x)(c-h)}\right)=0, \\
\mathbb{P} \mu=\mathbb{P} f(c)+A \bar{c} .
\end{gathered}
$$

As far as the function $f$ is concerned, we assume that $f \in \mathcal{C}^{2}\left(\mathbb{R}^{n}\right)$ and

$$
\begin{aligned}
& \left(\left(f^{\prime}(c) v, v\right)\right) \geq-\xi_{0}\|v\|^{2}, \quad \xi_{0} \geq 0, \forall c \in \mathbb{R}^{n}, \forall v \in T S, \\
& ((\overline{f(c)}, \bar{c})) \geq \alpha \int_{\Omega}\left(\bar{c}^{2} \cdot \bar{c}^{2}+\langle c\rangle^{2} \cdot \bar{c}^{2}\right) d x, \quad \alpha>0, \forall c \in S,
\end{aligned}
$$

where $\varphi^{2}=\left(\varphi_{i}^{2}\right)_{i}, \quad \varphi=\left(\varphi_{i}\right)_{i} \in \mathbb{R}^{n}$, and $f^{\prime}(c)=\left(\nabla f_{i}(c)\right)_{i}$ denotes the Jacobian matrix of $f, i=1, \ldots, n$.

Remark 2.1. In particular, assumptions (2.25) and (2.26) are satisfied by our proposed model (2.8)-(2.9). Indeed, we recall that

$$
f_{i}(c)=\frac{2}{n}\left[c_{i}\left(1-c_{i}\right)^{2}-c_{i}^{2}\left(1-c_{i}\right)\right]-\frac{1}{n} \sum_{i=1}^{n} \frac{\partial F}{\partial c_{i}},
$$

$i=1, \ldots, n$. We thus have

$$
\frac{\partial f_{i}}{\partial c_{i}}(c)=\frac{2}{n}\left[6 c_{i}^{2}-6 c_{i}+1\right]-\frac{2}{n^{2}}\left[6 c_{i}^{2}-6 c_{i}+1\right], \quad i=1, \ldots, n,
$$

and

$$
\frac{\partial f_{i}}{\partial c_{j}}(c)=-\frac{2}{n^{2}}\left[6 c_{j}^{2}-6 c_{j}+1\right], \quad i, j=1, \ldots, n, j \neq i .
$$

Therefore, the $i$-th component of $f^{\prime}(c) v$ reads

$$
\left(f^{\prime}(c) v\right)_{i}=\frac{2}{n}\left[6 c_{i}^{2}-6 c_{i}+1\right] v_{i}-\frac{2}{n^{2}} \sum_{j=1}^{n}\left(\left[6 c_{j}^{2}-6 c_{j}+1\right] v_{j}\right), \quad i=1, \ldots, n,
$$

hence

$$
\begin{aligned}
\left(\left(f^{\prime}(c) v, v\right)\right) & =\frac{2}{n} \int_{\Omega} \sum_{i=1}^{n}\left(\left[6 c_{i}^{2}-6 c_{i}+1\right] v_{i}^{2}\right) d x \\
& -\frac{2}{n^{2}} \int_{\Omega} \sum_{j=1}^{n}\left(\left[6 c_{j}^{2}-6 c_{j}+1\right] v_{j}\right) \sum_{i=1}^{n} v_{i} d x \\
& =\frac{2}{n} \int_{\Omega} \sum_{i=1}^{n}\left(\left[6 c_{i}^{2}-6 c_{i}+1\right] v_{i}^{2}\right) d x
\end{aligned}
$$


since $v \in T S$. It thus follows that

$$
\left(\left(f^{\prime}(c) v, v\right)\right) \geq-\sum_{i=1}^{n} \xi_{i}\left\|v_{i}\right\|^{2}
$$

where $\xi_{i}>0, i=1, \ldots, n$. On the other hand, we have

$$
\begin{aligned}
((\overline{f(c)}, \bar{c})) & =((f(c), \bar{c})) \\
& =\int_{\Omega} \sum_{i=1}^{n} f_{i}(c) \bar{c}_{i} d x \\
& =\frac{2}{n} \int_{\Omega} \sum_{i=1}^{n}\left[2 c_{i}^{3}-3 c_{i}^{2}+c_{i}\right] \bar{c}_{i} d x-\frac{1}{n} \int_{\Omega} \sum_{i=1}^{n} \frac{\partial F}{\partial c_{i}} \sum_{i=1}^{n} \bar{c}_{i} \\
& =\frac{2}{n} \int_{\Omega} \sum_{i=1}^{n}\left[2 c_{i}^{3}-3 c_{i}^{2}+c_{i}\right] \bar{c}_{i} d x \\
& =\frac{2}{n} \int_{\Omega} \sum_{i=1}^{n}\left[\overline{2 c_{i}^{3}-3 c_{i}^{2}+c_{i}}\right] \bar{c}_{i} d x
\end{aligned}
$$

for all $c \in S$, since $\bar{c} \in T S$. We then deduce from Remark 2.11 in [17] that

$$
((\overline{f(c)}, \bar{c})) \geq \alpha \int_{\Omega} \sum_{i=1}^{n}\left[\bar{c}_{i}^{4}+\left\langle c_{i}\right\rangle^{2} \bar{c}_{i}^{2}\right] d x, \quad \alpha>0 .
$$

We note that, for $v=\left(v_{i}\right)_{i} \in S$,

$$
\begin{gathered}
v \rightarrow\left(\|v-\langle v\rangle\|_{-1}^{2}+\langle v\rangle^{2}\right)^{\frac{1}{2}}, \\
v \rightarrow\left(\|v-\langle v\rangle\|^{2}+\langle v\rangle^{2}\right)^{\frac{1}{2}}, \\
v \rightarrow\left(\left\|A^{\frac{1}{2}} \bar{v}\right\|^{2}+\langle v\rangle^{2}\right)^{\frac{1}{2}},
\end{gathered}
$$

and

$$
v \rightarrow\left(\|A \bar{v}\|^{2}+\langle v\rangle^{2}\right)^{\frac{1}{2}}
$$

are norms in $L, H, V$, and $W$, respectively, which are equivalent to the usual ones. Here, $\|\cdot\|_{-1}=\left\|A^{-\frac{1}{2}} \cdot\right\|$.

Throughout this article, the same letter $\xi$ (and, sometimes, $\zeta$ and $\eta$ ) denotes constants which may vary from line to line, or even in a same line. Similarly, the same letter $Q$ denotes monotone increasing functions which may vary from line to line, or even in a same line. 


\section{A priori estimates}

We first multiply (2.23) by $\bar{c}$ and have

$$
\begin{aligned}
\frac{1}{2} \frac{d}{d t}\|\bar{c}\|_{-1}^{2} & =-((\overline{\mathbb{P} \mu}, \bar{c}))-\lambda_{0}\left(\left(A^{-1} \overline{\chi_{\Omega \backslash D}(x)(c-h)}, \bar{c}\right)\right) \\
& =-\left\|A^{\frac{1}{2}} \bar{c}\right\|^{2}-((\overline{f(c)}, \bar{c}))-\lambda_{0}\left(\left(\chi_{\Omega \backslash D}(x)(c-h), A^{-1} \bar{c}\right)\right) \\
& \leq-\left\|A^{\frac{1}{2}} \bar{c}\right\|^{2}-\alpha \int_{\Omega}\left(\bar{c}^{2} \cdot \bar{c}^{2}+\langle c\rangle^{2} \cdot \bar{c}^{2}\right) d x+\lambda_{0}(\|c\|+\|h\|)\|\bar{c}\| \\
& \leq-\left\|A^{\frac{1}{2}} \bar{c}\right\|^{2}-\alpha \int_{\Omega}\left(\bar{c}^{2} \cdot \bar{c}^{2}+\langle c\rangle^{2} \cdot \bar{c}^{2}\right) d x+\zeta\left(\|\bar{c}\|^{2}+\langle c\rangle^{2}\right)+\eta\left(\|h\|^{2}+1\right) \\
& \leq-\left\|A^{\frac{1}{2}} \bar{c}\right\|^{2}-\alpha \int_{\Omega}\left(\bar{c}^{2} \cdot \bar{c}^{2}+\langle c\rangle^{2} \cdot \bar{c}^{2}\right) d x+\frac{\alpha}{2} \int_{\Omega}\left(\bar{c}^{2} \cdot \bar{c}^{2}+\langle c\rangle^{2} \cdot \bar{c}^{2}\right) d x+\eta\left(\|h\|^{2}+1\right) \\
& \leq-\left\|A^{\frac{1}{2}} \bar{c}\right\|^{2}-\frac{\alpha}{2} \int_{\Omega}\left(\bar{c}^{2} \cdot \bar{c}^{2}+\langle c\rangle^{2} \cdot \bar{c}^{2}\right) d x+\eta\left(\|h\|^{2}+1\right),
\end{aligned}
$$

owing to (2.24) and (2.26), hence

$$
\frac{d}{d t}\|\bar{c}\|_{-1}^{2}+\left\|A^{\frac{1}{2}} \bar{c}\right\|^{2}+\alpha \int_{\Omega}\left(\bar{c}^{2} \cdot \bar{c}^{2}+\langle c\rangle^{2} \cdot \bar{c}^{2}\right) d x \leq \xi,
$$

which yields

$$
\frac{d}{d t}\|\bar{c}\|_{-1}^{2}+\xi\|\bar{c}\|_{-1}^{2} \leq \zeta, \quad \xi>0 .
$$

It thus follows from (3.2) and Gronwall's lemma that

$$
\|\bar{c}\|_{-1}^{2} \leq e^{-\xi t}\left\|\bar{c}_{0}\right\|_{-1}^{2}+\zeta, \quad \xi>0, \quad t \geq 0
$$

Let $B$ be a bounded subset of $\dot{H}^{-1}(\Omega)$ and $t_{0}$ be such that $\bar{c}_{0} \in B$ and $t \geq t_{0}$ implies $\bar{c}(t) \in \mathcal{B}_{0}$, where $\mathcal{B}_{0}=\left\{\phi \in \dot{H}^{-1}(\Omega),\|\phi\|_{-1}^{2} \leq 2 \zeta\right\}, \zeta$ being the constant in (3.3). We then deduce from (3.1) that, for $t \geq t_{0}$,

$$
\int_{t}^{t+r}\left\|A^{\frac{1}{2}} \bar{c}\right\|^{2} d s \leq \xi(r), \quad \int_{t}^{t+r} d s \int_{\Omega}\left(\bar{c}^{2} \cdot \bar{c}^{2}+\langle c\rangle^{2} \cdot \bar{c}^{2}\right) d x \leq \xi(r),
$$

for $r>0$ fixed.

We then multiply (2.23) by $A \bar{c}$ and find

$$
\begin{aligned}
\frac{1}{2} \frac{d}{d t}\|\bar{c}\|^{2} & =-((\mathbb{P} \mu, A \bar{c}))-\lambda_{0}\left(\left(\overline{\chi_{\Omega \backslash D}(x)(c-h)}, \bar{c}\right)\right) \\
& =-\|A \bar{c}\|^{2}-((f(c), A \bar{c}))-\lambda_{0}\left(\left(\chi_{\Omega \backslash D}(x)(c-h), \bar{c}\right)\right) \\
& \leq-\|A \bar{c}\|^{2}-\left(\left(f^{\prime}(c) A^{\frac{1}{2}} \bar{c}, A^{\frac{1}{2}} \bar{c}\right)\right)+\lambda_{0}(\|c\|+\|h\|)\|\bar{c}\| \\
& \leq-\|A \bar{c}\|^{2}+\xi_{0}\left\|A^{\frac{1}{2}} \bar{c}\right\|^{2}+\zeta \int_{\Omega}\left(\int_{\Omega} \bar{c}^{2} \cdot \bar{c}^{2}+\langle c\rangle^{2} \cdot \bar{c}^{2}\right) d x+\eta\|h\|^{2} \\
& \leq-\|A \bar{c}\|^{2}+\xi_{0}\left\|A^{\frac{1}{2}} \bar{c}\right\|^{2}+\zeta\left(\int_{\Omega}\left(\bar{c}^{2} \cdot \bar{c}^{2}+\langle c\rangle^{2} \cdot \bar{c}^{2}\right) d x+\|h\|^{2}+1\right),
\end{aligned}
$$

owing to (2.24) and (2.25), hence

$$
\frac{d}{d t}\|\bar{c}\|^{2}+\|A \bar{c}\|^{2} \leq \xi_{0}\left\|A^{\frac{1}{2}} \bar{c}\right\|+\zeta\left(\int_{\Omega}\left(\bar{c}^{2} \cdot \bar{c}^{2}+\langle c\rangle^{2} \cdot \bar{c}^{2}\right) d x+\|h\|^{2}+1\right) .
$$


It thus follows from (3.4), (3.5), and the uniform Gronwall's lemma that

$$
\|\bar{c}\|^{2} \leq \xi, \quad t \geq t_{0}+r,
$$

where the constant $\xi$ is independent of $\bar{c}_{0}$ and $t$, hence, employing (3.6) and integrating (3.1) and (3.5) between 0 and $t_{0}+r$,

$$
\|\bar{c}(t)\|^{2} \leq Q\left(\left\|\bar{c}_{0}\right\|\right), \quad t \geq 0 .
$$

Now, writing $c=\langle c\rangle+\bar{c}$ in $(2.20)$, we find

$$
\frac{d}{d t}\langle c\rangle+\frac{\lambda_{0}}{\operatorname{Vol}(\Omega)} \int_{\Omega \backslash D}(\langle c\rangle+\bar{c}-h) d x=0 .
$$

Therefore,

$$
\frac{d}{d t}\langle c\rangle+\xi\langle c\rangle=-\frac{\lambda_{0}}{\operatorname{Vol}(\Omega)} \int_{\Omega \backslash D}(\bar{c}-h) d x
$$

where $\xi=\frac{\lambda_{0} \operatorname{Vol}(\Omega \backslash D)}{\operatorname{Vol}(\Omega)}$, hence

$$
\frac{d}{d t}\left(e^{\xi t}\langle c\rangle\right)=-\frac{\lambda_{0}}{\operatorname{Vol}(\Omega)} e^{\xi t} \int_{\Omega \backslash D}(\bar{c}-h) d x
$$

and

$$
\langle c\rangle=e^{-\xi t}\left\langle c_{0}\right\rangle-\frac{\lambda_{0}}{\operatorname{Vol}(\Omega)} e^{-\xi t} \int_{0}^{t} e^{\xi s} \int_{\Omega \backslash D}(\bar{c}-h) d x d s .
$$

Thus,

$$
|\langle c\rangle| \leq e^{-\xi t}\left|\left\langle c_{0}\right\rangle\right|+\zeta e^{-\xi t} \int_{0}^{t} e^{\xi s}(\|\bar{c}\|+\|h\|) d s, \quad \forall t \geq 0,
$$

where $\zeta=\frac{\lambda_{0}}{(\operatorname{Vol}(\Omega))^{\frac{1}{2}}}$. Here,

$$
\begin{aligned}
\zeta e^{-\xi t} \int_{0}^{t} e^{\xi s}\|h\| d s & \leq \zeta e^{-\xi t}\|h\| e^{\xi t} \\
& \leq \zeta\|h\| \\
& \leq \eta
\end{aligned}
$$

Furthermore, for $t \geq t_{0}+r$,

$$
\begin{aligned}
\zeta e^{-\xi t} \int_{0}^{t} e^{\xi s}\|\bar{c}\| d s & =\zeta e^{-\xi t} \int_{0}^{t_{0}+r} e^{\xi s}\|\bar{c}\| d s+\zeta e^{-\xi t} \int_{t_{0}+r}^{t} e^{\xi s}\|\bar{c}\| d s \\
& \leq Q\left(\left\|\bar{c}_{0}\right\|\right) e^{-\xi t} e^{\xi\left(t_{0}+r\right)}+\zeta e^{-\xi t} \int_{t_{0}+r}^{t} e^{\xi s}\|\bar{c}\| d s \\
& \leq Q\left(\left\|\bar{c}_{0}\right\|\right) e^{-\xi t}+\zeta e^{-\xi t} \int_{t_{0}+r}^{t} e^{\xi s}\|\bar{c}\| d s \\
& \leq Q\left(\left\|\bar{c}_{0}\right\|\right) e^{-\xi t}+\eta e^{-\xi t}\left(e^{\xi t}-e^{\xi\left(t_{0}+r\right)}\right) \\
& \leq Q\left(\left\|\bar{c}_{0}\right\|\right) e^{-\xi t}+\zeta
\end{aligned}
$$

where we have used (3.6) (resp., (3.7)) to estimate the first (resp., the second) integral in the right-hand side of the first equality. Finally, we obtain

$$
|\langle c\rangle| \leq\left(Q\left(\left\|\bar{c}_{0}\right\|\right)+\left|\left\langle c_{0}\right\rangle\right|\right) e^{-\xi t}+\zeta, \quad \forall t \geq 0,
$$


where $\xi$ and $\zeta$ are two constants which are nonnegative and independent of $t$ and $c_{0}$.

We finally multiply $(2.18)$ by $A^{2} \bar{c}$ to obtain, noting that

$$
\left(\left(A \overline{\mathbb{P}} \mu, A^{2} \bar{c}\right)\right)=\left(\left(\mathbb{P} \mu, A^{3} \bar{c}\right)\right)=\left(\left(\mu, A^{3} \bar{c}\right)\right),
$$

the differential equalities

$$
\begin{aligned}
\frac{1}{2} \frac{d}{d t}\|A \bar{c}\|^{2} & =-\left(\left(\mu, A^{3} \bar{c}\right)\right)-\lambda_{0}\left(\left(\chi_{\Omega \backslash D}(x)(c-h), A^{2} \bar{c}\right)\right) \\
& =-\left\|A^{2} \bar{c}\right\|^{2}-\left(\left(A \overline{f(c)}, A^{2} \bar{c}\right)\right)-\lambda_{0}\left(\left(\chi_{\Omega \backslash D}(x)(c-h), A^{2} \bar{c}\right)\right) .
\end{aligned}
$$

Using the fact that

$$
\Delta f(c)=f^{\prime}(c) \Delta c+f^{\prime \prime}(c)|\nabla c|^{2},
$$

we have (see, e.g., [46])

$$
\begin{aligned}
\left|\left(\left(A \overline{f(c)}, A^{2} \bar{c}\right)\right)\right| & =\left|\left(\left(-\Delta f(c), A^{2} \bar{c}\right)\right)\right| \\
& \leq \xi\|\Delta f(c)\|^{2}+\frac{1}{4}\left\|A^{2} \bar{c}\right\|^{2} \\
& \leq \frac{1}{2}\left\|A^{2} \bar{c}\right\|^{2}+\xi .
\end{aligned}
$$

Furthermore,

$$
\begin{aligned}
\lambda_{0}\left|\left(\left(\chi_{\Omega \backslash D}(x)(c-h), A^{2} \bar{c}\right)\right)\right| & \leq \xi\|c-h\|\left\|A^{2} \bar{c}\right\| \\
& \leq \xi\left(\|\bar{c}\|^{2}+\langle c\rangle^{2}\right)+\frac{1}{2}\left\|A^{2} \bar{c}\right\|^{2}+\zeta\|h\|^{2} .
\end{aligned}
$$

We finally deduce from (3.9), (3.10), and (3.11) that

$$
\frac{d}{d t}\|A \bar{c}\|^{2} \leq \xi\left(\|\bar{c}\|^{2}+\langle c\rangle^{2}\right)+\zeta .
$$

Note that, integrating (3.5) over $(t, t+r)$, we have, for $t \geq t_{0}+r$,

$$
\int_{t}^{t+r}\|A \bar{c}\|^{2} d x \leq \xi(r),
$$

owing to (3.6). Furthermore, owing to (2.20), we have

$$
\begin{aligned}
\frac{d}{d t}\langle c\rangle^{2} & =2\langle c\rangle \frac{d}{d t}\langle c\rangle \\
& \leq 2 \lambda_{0}|\langle c\rangle|\left|\int_{\Omega \backslash D}(c-h) d x\right| \\
& \leq \xi\left(\|\bar{c}\|^{2}+(\langle c\rangle)^{2}\right)+\zeta\left(\|h\|^{2}+1\right)
\end{aligned}
$$

and, owing to (3.6) and (3.8), $\exists t_{1}$ such that, $\forall t \geq t_{1}$,

$$
|\langle c\rangle| \leq \xi \quad \text { and } \quad\|\bar{c}\|^{2} \leq \xi .
$$

We finally deduce from (3.6), (3.8), (3.12), (3.13), (3.14), (3.15), and the uniform Gronwall's lemma that

$$
\|c\|_{W} \leq \xi, \quad t \geq t_{1}+r
$$

where $r>0$ is fixed. 


\section{Well-posedness and existence of the global attractor}

We have the

Theorem 4.1. We assume that $c_{0} \in H$. Then, (2.18)-(2.19) possesses a unique solution $c$ such that

$$
c \in L^{\infty}([0, T], H) \cap L^{2}([0, T], W) \cap L^{4}\left([0, T],\left(L^{4}(\Omega)\right)^{n} \cap S\right), \quad \forall T>0 .
$$

Proof. We first denote by $E_{m}$ the space

$$
E_{m}=\operatorname{span}\left\{N_{1}, N_{2}, \ldots, N_{m}\right\}
$$

and by $P_{m}$ the orthogonal projection from $\dot{V}$ onto $E_{m}$,

$$
P_{m} h=\sum_{j=1}^{m}\left(\left(h, N_{j}\right)\right) N_{j} .
$$

The variational formulation of the problem reads: Find $c_{m}:[0, T] \rightarrow\left\langle c_{m}\right\rangle+E_{m}$, $c_{m}(t)=\left\langle c_{m}(t)\right\rangle+\bar{c}_{m}(t)=\left\langle c_{m}(t)\right\rangle+\sum_{j=1}^{m} c_{m_{j}}(t) N_{j}$, such that

$$
\begin{gathered}
\frac{d}{d t}\left(\left(A^{-1} \sum_{i=1}^{m} \bar{c}_{m}(t) N_{i}, N_{j}\right)\right)-\left(\left(\sum_{i=1}^{m} \bar{c}_{m_{i}}(t) A^{\frac{1}{2}} N_{i}, A^{\frac{1}{2}} N_{j}\right)\right)+\left(\left(\overline{\mathbb{P} f\left(\left\langle c_{m}\right\rangle+\bar{c}_{m}\right)}, N_{j}\right)\right) \\
+\lambda_{0}\left(\left(A^{-1}\left(\overline{\chi_{\Omega \backslash D}(x)\left(\left\langle c_{m}\right\rangle+\bar{c}_{m}-h\right)}\right), N_{j}\right)\right)=0, \quad j=1, \ldots, m \\
\frac{d}{d t}\left\langle c_{m}\right\rangle+\xi\left\langle c_{m}\right\rangle=-\frac{\lambda_{0}}{\operatorname{Vol}(\Omega)} \int_{\Omega \backslash D}\left(\bar{c}_{m}-h\right) d x \\
\bar{c}_{m}(0)=P_{m} c_{0} \\
\left\langle c_{m}(0)\right\rangle=\left\langle c_{0}\right\rangle
\end{gathered}
$$

We can rewrite (4.1) and (4.3) as

$$
\begin{aligned}
\frac{\partial A^{-1} \bar{c}_{m}}{\partial t} & +\overline{\mathbb{P} f\left(\left\langle c_{m}\right\rangle+\bar{c}_{m}\right)}+A \bar{c}_{m}+\lambda_{0} A^{-1}\left(\overline{\chi_{\Omega \backslash D}(x)\left(\left\langle c_{m}\right\rangle+\bar{c}_{m}-h\right)}\right) \\
& =0, \quad \text { in } E_{m}^{\prime},
\end{aligned}
$$

and we finally rewrite equation (4.1) as follows:

$$
M^{-1} \frac{d Y}{d t}+M Y+H(Y)=0
$$

where $M=\left(\left(A N_{i}, N_{j}\right)\right)_{i, j=1, \ldots, m}, Y=\left(\begin{array}{c}c_{m_{1}} \\ \cdot \\ \cdot \\ \cdot \\ c_{m_{m}}\end{array}\right)$, and 


$$
H(Y)=\left(\begin{array}{c}
\left(\left(\overline{\mathbb{P} f\left(\left\langle c_{m}\right\rangle+\bar{c}_{m}\right)}+\lambda_{0} A^{-1}\left(\overline{\chi_{\Omega \backslash D}\left(\mathbb{P}\left(\left\langle c_{m}\right\rangle+\bar{c}_{m}-h\right)\right)}\right), N_{1}\right)\right) \\
\cdot \\
\cdot \\
\left(\left(\overline{\mathbb{P} f\left(\left\langle c_{m}\right\rangle+\bar{c}_{m}\right)}+\lambda_{0} A^{-1}\left(\frac{\cdot}{\chi_{\Omega \backslash D}\left(\mathbb{P}\left(\left\langle c_{m}\right\rangle+\bar{c}_{m}-h\right)\right)}\right), N_{m}\right)\right)
\end{array}\right)
$$

noting that $\left\langle c_{m}\right\rangle$ can be expressed as a function of $\bar{c}_{m}$ by solving (4.2). The matrix $M$ is invertible and positive definite and $H(Y)$ depends continuously on $Y$. Applying Cauchy's theorem, we find that there exists a time $t_{m} \in(0, T)$ and a solution $Y$ to

$$
\frac{d Y}{d t}+M^{2} Y+M H(Y)=0
$$

on the time interval $\left[0, t_{m}\left[\right.\right.$. Having $\bar{c}_{m}$, we then deduce $\left\langle c_{m}\right\rangle$ (from (4.2)).

It follows from the a priori estimates derived in the previous section for the solution $c_{m}(t)\left(c(t)\right.$ being replaced by $\left.c_{m}(t)\right)$ that any local solution to $(2.18)-(2.19)$ is actually a global solution defined on the whole interval $[0, T]$. It then follows from the a priori estimates that, up to a subsequence which we do not relabel,

$$
\begin{gathered}
c_{m} \rightarrow c \text { weakly in } L^{2}(0, T, W), \\
\frac{\partial c_{m}}{\partial t} \rightarrow \frac{\partial c}{\partial t} \text { weakly in } L^{\frac{4}{3}}\left(0, T, W^{-2, \frac{4}{3}}(\Omega)\right),
\end{gathered}
$$

as $m \rightarrow \infty$. It follows from (4.7), (4.8), and the Aubin-Lions compactness theorem that

$$
c_{m} \rightarrow c \text { strongly in } L^{\frac{4}{3}}\left(0, T,\left(L^{\frac{4}{3}}(\Omega)\right)^{n} \cap S\right)
$$

and $c_{m}(t, x) \rightarrow c(x, t)$ a.e. $(t, x) \in[0, T] \times \Omega$. Moreover, since $f$ is a continuous,

$$
f\left(c_{m}(t, x)\right) \rightarrow f(c(t, x)) \text { a.e. }
$$

and, since $f\left(c_{m}\right)$ is bounded in $L^{\frac{4}{3}}\left(\Omega_{T}\right), \Omega_{T}=(0, T) \times \Omega$,

$$
f\left(c_{m}\right) \rightarrow f(c) \text { weakly in } L^{\frac{4}{3}}\left(\Omega_{T}\right) \cap T S,
$$

owing to the weak dominated convergence theorem. Finally, we deduce that $A^{-1} \frac{\partial \bar{c}_{m}}{\partial t} \rightarrow$ $A^{-1} \frac{\partial \bar{c}}{\partial t}$ weakly in $L^{\frac{4}{3}}\left(\Omega_{T}\right)$. Thus, passing to the limit in (4.5), we obtain

$$
\frac{\partial A^{-1} \bar{c}}{\partial t}+\overline{\mathbb{P} f(c)}+A \bar{c}+\lambda_{0} A^{-1}\left(\overline{\chi_{\Omega \backslash D}(x)(\mathbb{P} c-\mathbb{P} h)}\right)=0, \quad \text { in } L^{\frac{4}{3}}\left(\Omega_{T}\right) \cap T S .
$$

Finally, we easily pass to the limit in (4.2), at least in a weak sense, i.e., solving explicitly this ODE.

Let now $c_{1}$ and $c_{2}$ be two solutions to (2.18)-(2.19) with initial data $c_{0,1}$ and $c_{0,2}$, respectively. We set $c=c_{1}-c_{2}, c_{0}=c_{0,1}-c_{0,2}$, and $\mu=\mu_{1}-\mu_{2}$ and have

$$
\begin{gathered}
\frac{\partial c}{\partial t}+A \overline{\mathbb{P} \mu}+\lambda_{0} \chi_{\Omega \backslash D}(x) c=0, \\
\mathbb{P} \mu=\mathbb{P}\left(f\left(c_{1}\right)-f\left(c_{2}\right)\right)+A \bar{c}, \\
\left.c\right|_{t=0}=c_{0}=c_{0,1}-c_{0,2} .
\end{gathered}
$$


Integrating (4.11) over $\Omega$, we obtain

$$
\frac{d}{d t}\langle c\rangle=-\frac{\lambda_{0}}{\operatorname{Vol}(\Omega)} \int_{\Omega \backslash D} c d x .
$$

We can thus rewrite the problem as

$$
\begin{aligned}
& \frac{\partial \bar{c}}{\partial t}+A \overline{\mathbb{P} \mu}+\lambda_{0} \overline{\chi_{\Omega \backslash D}(x) c}=0, \\
& \mathbb{P} \mu=\mathbb{P}\left(f\left(c_{1}\right)-f\left(c_{2}\right)\right)+A \bar{c},
\end{aligned}
$$

or else

$$
\begin{gathered}
\frac{\partial A^{-1} \bar{c}}{\partial t}+\overline{\mathbb{P} \mu}+\lambda_{0} A^{-1} \overline{\chi_{\Omega \backslash D}(x) c}=0, \\
\mathbb{P} \mu=\mathbb{P}\left(f\left(c_{1}\right)-f\left(c_{2}\right)\right)+A \bar{c} .
\end{gathered}
$$

We multiply (4.17) by $\bar{c}$ and find

$$
\begin{aligned}
& \frac{1}{2} \frac{d}{d t}\|\bar{c}\|_{-1}^{2}=-((\mu, \bar{c}))-\lambda_{0}\left(\left(A^{-1} \overline{\chi_{\Omega \backslash D}(x) c}, \bar{c}\right)\right) \\
& \quad=-\left(\left(f\left(c_{1}\right)-f\left(c_{2}\right), c\right)\right)+\left(\left(f\left(c_{1}\right)-f\left(c_{2}\right),\langle c\rangle\right)\right) \\
& \quad-\left\|A^{\frac{1}{2}} \bar{c}\right\|^{2}-\lambda_{0}\left(\left(\overline{\chi_{\Omega \backslash D}(x) c}, A^{-1} \bar{c}\right)\right) \\
& \quad \leq \xi_{0}\|c\|^{2}+\left(\left(f\left(c_{1}\right)-f\left(c_{2}\right),\langle c\rangle\right)\right)-\left\|A^{\frac{1}{2}} \bar{c}\right\|^{2}-\lambda_{0}\left(\left(\chi_{\Omega \backslash D}(x) c, A^{-1} \bar{c}\right)\right) \\
& \quad \leq \xi_{0}\|c\|^{2}+\left|\left(\left(f\left(c_{1}\right)-f\left(c_{2}\right),\langle c\rangle\right)\right)\right|-\left\|A^{\frac{1}{2}} \bar{c}\right\|^{2}+\xi\|c\|\|\bar{c}\|_{-1} \\
& \quad \leq \xi\left(\|\bar{c}\|^{2}+\langle c\rangle^{2}\right)+\zeta\|\bar{c}\|_{-1}^{2}-\left\|A^{\frac{1}{2}} \bar{c}\right\|^{2}+\left|\left(\left(f\left(c_{1}\right)-f\left(c_{2}\right),\langle c\rangle\right)\right)\right|,
\end{aligned}
$$

owing to (2.25) and (4.18). We note that, owing also to (2.25),

$$
\begin{aligned}
\mid\left(\left(f\left(c_{1}\right)-f\left(c_{2},\langle c\rangle\right)\right) \mid\right. & =\left|\langle c\rangle \int_{\Omega} c \int_{0}^{1} f^{\prime}\left(c_{1}+s\left(c_{2}-c_{1}\right)\right) d s d x\right| \\
& \leq \xi|\langle c\rangle| \int_{\Omega}\left(\left|c_{1}\right|^{2}+\left|c_{2}\right|^{2}+1\right)|c| d x \\
& \leq \xi|\langle c\rangle|\left(\left\|c_{1}\right\|_{\left(L^{4}(\Omega)\right)^{n}}^{2}+\left\|c_{2}\right\|_{\left(L^{4}(\Omega)\right)^{n}}^{2}+1\right)\|c\| \\
& \leq \xi\left(\left\|c_{1}\right\|_{\left(L^{4}(\Omega)\right)^{n} \cap S}^{2}+\left\|c_{2}\right\|_{\left(L^{4}(\Omega)\right)^{n} \cap S}^{2}+1\right)\left(|\langle c\rangle|^{2}+\|\bar{c}\|^{2}\right)
\end{aligned}
$$

hence

$$
\begin{array}{r}
\frac{1}{2} \frac{d}{d t}\|\bar{c}\|_{-1}^{2}+\left\|A^{\frac{1}{2}} \bar{c}\right\|^{2} \leq \xi\left(\left\|c_{1}\right\|_{\left(L^{4}(\Omega)\right)^{n} \cap S}^{2}+\left\|c_{2}\right\|_{\left(L^{4}(\Omega)\right)^{n} \cap S}^{2}\right. \\
+1)\left(|\langle c\rangle|^{2}+\|\bar{c}\|^{2}\right)+\zeta\|\bar{c}\|_{-1}^{2} .
\end{array}
$$

Noting then that

$$
\begin{aligned}
\frac{1}{2} \frac{d}{d t}|\langle c\rangle|^{2} & \leq|\langle c\rangle|\left|\frac{d}{d t}\langle c\rangle\right| \\
& \leq \xi|\langle c\rangle|\left|\int_{\Omega \backslash D} c d x\right| \\
& \leq \xi|\langle c\rangle|\|c\| \\
& \leq \xi\left(\langle c\rangle^{2}+\|\bar{c}\|^{2}\right)
\end{aligned}
$$

and recalling the interpolation inequality

$$
\|\bar{c}\|^{2} \leq \xi\|\bar{c}\|_{-1}\left\|A^{\frac{1}{2}} \bar{c}\right\|,
$$


it follows that

$$
\begin{gathered}
\frac{d}{d t}\left(\|\bar{c}\|_{-1}^{2}+|\langle c\rangle|^{2}\right)+\left\|A^{\frac{1}{2}} \bar{c}\right\|^{2} \leq \xi( \\
+1)\left(|\langle c\rangle|^{2}\left\|_{\left(L^{4}(\Omega)\right)^{n} \cap S}^{4}+\right\| \bar{c} \|_{-1}^{2}\right) .
\end{gathered}
$$

We thus deduce from (4.21) and Gronwall's lemma that

$$
\left\|c_{1}(t)-c_{2}(t)\right\|_{L} \leq Q\left(T,\left\|c_{0,1}\right\|,\left\|c_{0,2}\right\|\right)\left\|c_{0,1}-c_{0,2}\right\|_{L}, \quad 0 \leq t \leq T,
$$

hence the uniqueness, as well as the continuous dependence with respect to the initial data in the $L$-norm.

It follows from Theorem 4.1 that we have the continuous (with respect to the $L^{-}$ norm) semigroup

$$
S(t): H \rightarrow H, \quad c_{0} \mapsto c(t), \quad t \geq 0
$$

(i.e., $S(0)=I, S(t+s)=S(t) \circ S(s), t, s \geq 0)$.

It then follows from (3.16) that $S(t)$ possesses a bounded absorbing set $\mathcal{B}_{1}$ which is compact in $H$ and bounded in $W$. We thus deduce from standard results (see, e.g., $[41,46])$ the following theorem.

Theorem 4.2. The semigroup $S(t)$ possesses the connected global attractor $\mathcal{A}$ such that $\mathcal{A}$ is compact in $H$ and bounded in $W$.

\section{$5 \quad$ Existence of exponential attractors}

Let $c_{1}$ and $c_{2}$ be two solutions to (2.18)-(2.19) with initial data $c_{0,1}$ and $c_{0,2}$, respectively. We set $c=c_{1}-c_{2}, c_{0}=c_{0,1}-c_{0,2}$, and $\mu=\mu_{1}-\mu_{2}$ and have

$$
\begin{gathered}
\frac{\partial c}{\partial t}+A \overline{\mathbb{P} \mu}+\lambda_{0} \chi_{\Omega \backslash D}(x) c=0, \\
\mathbb{P} \mu=\mathbb{P}\left(f\left(c_{1}\right)-f\left(c_{2}\right)\right)+A \bar{c}, \\
\left.c\right|_{t=0}=c_{0} .
\end{gathered}
$$

Furthermore, it is sufficient here to take initial data belonging to the bounded absorbing set $\mathcal{B}_{1}$ defined in the previous section.

We multiply (5.1) by $t c$ to find, owing to (2.25) and (5.2),

$$
\begin{aligned}
\frac{1}{2} \frac{d}{d t}\left(t\|c\|^{2}\right)+\lambda_{0} t\left\|\chi_{\Omega \backslash D}(x) c\right\|^{2} & =\frac{1}{2}\|c\|^{2}-t\|A \bar{c}\|^{2}-t\left(\left(A^{\frac{1}{2}}\left(\overline{f\left(c_{1}\right)-f\left(c_{2}\right)}\right), A^{\frac{1}{2}} \bar{c}\right)\right) \\
& \leq \frac{1}{2}\|c\|^{2}+\xi_{0} t\left\|A^{\frac{1}{2}} \bar{c}\right\|^{2}
\end{aligned}
$$

which yields

$$
\frac{d}{d t}\left(t\|c\|^{2}\right) \leq\|c\|^{2}+\xi t\left\|A^{\frac{1}{2}} \bar{c}\right\|^{2} .
$$

Integrating (5.4) between 0 and $t$, we obtain

$$
\|c\|^{2} \leq \xi \frac{1+t}{t} \int_{0}^{t}\|c\|_{V}^{2} d s, \quad t>0 .
$$


Noting that (4.21) is equivalent to

$$
\frac{d}{d t}\left(\|\bar{c}\|_{-1}^{2}+|\langle c\rangle|^{2}\right)+\|c\|_{V}^{2} \leq \xi\left(\left\|c_{1}\right\|_{\left(L^{4}(\Omega)\right)^{n} \cap S}^{4}+\left\|c_{2}\right\|_{\left(L^{4}(\Omega)\right)^{n} \cap S}^{4}+1\right)\left(|\langle c\rangle|^{2}+\|\bar{c}\|_{-1}^{2}\right),
$$

it follows from (3.1), (4.22), and (5.6) that

$$
\int_{0}^{t}\|c\|_{V}^{2} d s \leq \xi e^{\zeta t}\|c\|_{L}^{2}
$$

We then deduce from (5.4) and (5.7) that

$$
t\|c\|_{H}^{2} \leq \xi e^{\zeta t}\|c\|_{L}^{2}, \quad t>0 .
$$

Next, we derive a Hölder (both with respect to space and time) estimate. Actually, owing to (4.22), it suffices to prove the Hölder continuity with respect to time. We have

$$
\begin{aligned}
\left\|c\left(t_{1}\right)-c\left(t_{2}\right)\right\|_{L} & =\left\|\int_{t_{1}}^{t_{2}} \frac{\partial c}{\partial t} d \tau\right\|_{L} \leq\left|\int_{t_{1}}^{t_{2}}\left\|\frac{\partial c}{\partial t}\right\|_{L} d \tau\right| \\
& \leq\left|t_{1}-t_{2}\right|^{\frac{1}{2}}\left|\int_{t_{1}}^{t_{2}}\left\|\frac{\partial c}{\partial t}\right\|_{L}^{2} d \tau\right|^{\frac{1}{2}} .
\end{aligned}
$$

Multiplying (2.23) by $\frac{\partial \bar{c}}{\partial t}$, we find

$$
\begin{aligned}
\left\|\frac{\partial \bar{c}}{\partial t}\right\|_{-1}^{2} & =-\left(\left(f(c), \frac{\partial \bar{c}}{\partial t}\right)\right)-\frac{1}{2} \frac{d}{d t}\left\|A^{\frac{1}{2}} \bar{c}\right\|^{2}+\lambda_{0}\left(\left(\chi_{\Omega \backslash D}(x)(h-c), A^{-1} \frac{\partial \bar{c}}{\partial t}\right)\right) \\
& \leq\left\|A^{\frac{1}{2}} \overline{f(c)}\right\|\left\|\frac{\partial \bar{c}}{\partial t}\right\|_{-1}-\frac{1}{2} \frac{d}{d t}\left\|A^{\frac{1}{2}} \bar{c}\right\|^{2}+\lambda_{0}\|c-h\|\left\|\frac{\partial \bar{c}}{\partial t}\right\|_{-1},
\end{aligned}
$$

owing to (2.24), hence

$$
\frac{d}{d t}\left\|A^{\frac{1}{2}} \bar{c}\right\|^{2}+\left\|\frac{\partial \bar{c}}{\partial t}\right\|_{-1}^{2} \leq \xi\|c\|_{V}^{2}+\zeta
$$

owing to $(2.25)$ and the continuous embedding $H^{2}(\Omega) \subset \mathcal{C}(\bar{\Omega})$. Noting that

$$
\left\langle\frac{\partial c}{\partial t}\right\rangle^{2} \leq \xi\|c\|^{2}+\zeta
$$

owing to (2.20), we then deduce from (3.16), (5.10), and (5.11) that

$$
\int_{t_{1}}^{t_{2}}\left\|\frac{\partial c}{\partial t}\right\|_{L}^{2} d \tau \leq \xi
$$

where the constant $\xi$ depends on $\mathcal{B}_{1}$ and $T$ such that $t_{1}, t_{2} \in[0, T]$, so that

$$
\left\|c\left(t_{1}\right)-c\left(t_{2}\right)\right\|_{L} \leq \xi\left|t_{1}-t_{2}\right|^{\frac{1}{2}}
$$

where the constant $\xi$ depends on $\mathcal{B}_{1}$ and $T$ such that $t_{1}, t_{2} \in[0, T]$.

We finally deduce from (4.22), (5.8), and (5.13) the following result (see, e.g., [22, 23]).

Theorem 5.1. The semigroup $S(t)$ possesses an exponential attractor $\mathcal{M} \subset \mathcal{B}_{1}$, i.e., 
(i) $\mathcal{M}$ is compact in $L$;

(ii) $\mathcal{M}$ is positively invariant, $S(t) \mathcal{M} \subset \mathcal{M}, \forall t \geq 0$;

(iii) $\mathcal{M}$ has finite fractal dimension in $L$;

(iv) $\mathcal{M}$ attracts exponentially fast the bounded subsets of $H$,

$$
\begin{gathered}
\forall B \subset H \text { bounded, } \operatorname{dist}_{L}(S(t) B, \mathcal{M}) \leq Q\left(\|B\|_{H}\right) e^{-\xi t}, \\
\xi>0, \quad t \geq 0,
\end{gathered}
$$

where the constant $\xi$ is independent of $B$ and dist ${ }_{L}$ denotes the Hausdorff semidistance between sets defined by

$$
\operatorname{dist}_{L}(A, B)=\sup _{a \in A} \inf _{b \in B}\|a-b\|_{L}
$$

Remark 5.2. Setting $\tilde{\mathcal{M}}=S(1) \mathcal{M}$, we can prove that $\tilde{\mathcal{M}}$ is an exponential attractor for $S(t)$, but now in the topology of $H$ (see, e.g., [24]).

Since $\mathcal{M}$ (or $\tilde{\mathcal{M}}$ ) is a compact attracting set, we deduce from Theorem 5.1 the following corollary.

Corollary 5.3. The semigroup $S(t)$ possesses the finite-dimensional global attractor $\mathcal{A} \subset \mathcal{B}_{1}$.

Remark 5.4. We can more generally consider a nonlinear term $F$ of the form

$$
F(v)=\sum_{i=1}^{n} \sum_{k=0}^{2 p+2} a_{k} v_{i}^{k}, \quad a_{2 p+2}>0, \quad v=\left(v_{i}\right)_{i} \in S,
$$

but, now, assumption (2.26) is replaced by

$$
((\overline{f(c)}, \bar{c})) \geq \alpha \int_{\Omega} \sum_{i=1}^{n} \sum_{k=0}^{p} \bar{c}_{i}^{2 p+2-2 k}\langle c\rangle^{2 k} d x, \quad \alpha>0, \quad c=\left(c_{i}\right)_{i} \in S .
$$

Indeed, since $F(c)=\sum_{i=1}^{n} \sum_{k=0}^{2 p+2} a_{k} c_{i}^{k}, a_{2 p+2}>0$, we have

$$
\frac{\partial F(c)}{\partial c_{i}}=\sum_{k=0}^{2 p+1}(k+1) a_{k+1} c_{i}^{k}, \quad i=1, \ldots, n,
$$

hence

$$
\frac{\partial^{2} F(c)}{\partial c_{i}^{2}} \geq-\xi_{i}, \quad \xi_{i} \geq 0
$$

$i=1, \ldots, n$, and, owing to Remark 2.1,

$$
\begin{aligned}
\left(\left(f^{\prime}(c) v, v\right)\right) & =\int_{\Omega} \sum_{i=1}^{n} \frac{\partial^{2} F(c)}{\partial c_{i}^{2}} v_{i}^{2} d x \\
& \geq-\sum_{i=1}^{n} \xi_{i}\left\|v_{i}\right\|^{2}, \quad \xi_{i} \geq 0
\end{aligned}
$$


for all $v \in T S$. Furthermore, it follows from Remark 2.11 in [17] that

$$
\left(\left(\frac{\overline{\partial F(c)}}{\partial c_{i}}, \bar{c}_{i}\right)\right) \geq \alpha \int_{\Omega} \sum_{k=0}^{p} \bar{c}_{i}^{2 p+2-2 k}\langle c\rangle^{2 k} d x, \quad \alpha>0, c \in S,
$$

$i=1, \ldots, n$, and, owing to Remark 2.1,

$$
\begin{aligned}
((\overline{f(c)}, \bar{c})) & =\int_{\Omega} \sum_{i=1}^{n} \frac{\overline{\partial F(c)}}{\partial c_{i}} \bar{c}_{i} d x \\
& \geq \alpha \int_{\Omega} \sum_{i=1}^{n} \sum_{k=0}^{p} \bar{c}_{i}^{2 p+2-2 k}\langle c\rangle^{2 k} d x, \quad \alpha>0
\end{aligned}
$$

for all $c \in S$.

Remark 5.5. We studied the (global) dynamics of the system when $\lambda_{0}$ is constant and $\varepsilon=1$. More generally, all constants here and in the previous sections depend on $\lambda_{0}$ and $\varepsilon$ and grow as these quantities go to $+\infty$ and 0 , respectively. In particular, the dependence on $\lambda_{0}$ is an important issue in view of inpainting applications (see [4]) and a natural question is whether one can have an upper bound on the dimension of the global attractor which is independent of this quantity (here, the upper bound that we obtain explodes as $\lambda_{0}$ goes to $+\infty$ ). A natural (but involved, see, e.g., [46]) problem would be to find a lower bound on this dimension (possibly, in terms of $\lambda_{0}$ ). This will be investigated elsewhere.

\section{Algebraic consistency with the two-phase model}

In order to ensure the "physical" constraints of our proposed model, the model must (at least, see below) satisfy two properties:

(i) When phases $\left(i_{1}, i_{2}, i_{3}, \ldots, i_{n-2}\right) \in\{1, \ldots, n\}$ are not present, the $n$-phase free energy is equal to the one of the two-phase model.

(ii) When phases $\left(i_{1}, i_{2}, i_{3}, \ldots, i_{n-2}\right) \in\{1, \ldots, n\}$ are not present at the initial time (i.e., $c_{i_{j}}(0)=0$ and $\left.h_{i_{j}}=0, j=1, \ldots, n-2\right)$, these phases do not appear artificially during the evolution of the system.

In that case, we say that the model is algebraically consistent with the two-phase model (see [7]). Of course, the model should also be consistent with the $k$-phase model, $k<n$, but we have not been able to prove this more general property here.

Theorem 6.1. Model (2.8)-(2.9) is algebraically consistent with the diphasic system.

Proof. We first recall that the total free energy of (2.8)-(2.9) ( $n$-phase model) can be written as follows:

$$
\mathcal{F}_{n}\left(\left(c_{i}\right)_{i}\right)=\int_{\Omega}\left[\frac{1}{n} \sum_{i=1}^{n}\left(c_{i}^{2}\left(1-c_{i}\right)^{2}\right)+\frac{\varepsilon^{2}}{2} \sum_{i=1}^{n}\left|\nabla c_{i}\right|^{2}\right] d x+\frac{\lambda_{0}}{2} \sum_{i=1}^{n} \int_{\Omega \backslash D}\left(c_{i}-h_{i}\right)^{2} d x .
$$

We assume that $c_{i}=c, c_{j}=1-c$, and $c_{k}=h_{k}=0$, for $i$ and $j$ fixed in $\{1,2, \ldots, n\}$ and $k=1, \ldots, n, k \neq i, j$. We set $h_{i}=h$ (i.e., $\left.h_{j}=1-h\right)$ and $\tilde{c}=(0, \ldots, 0, c, 0, . ., 0,1-$ $c, 0, . ., 0) \in \mathbb{R}^{n}$. Then, the total free energy can be rewritten as

$$
\mathcal{F}_{n}(\tilde{c})=\int_{\Omega}\left[\frac{2}{n}\left(c^{2}(1-c)^{2}\right)+\varepsilon^{2}|\nabla c|^{2}\right] d x+\lambda_{0} \int_{\Omega \backslash D}(c-h)^{2} d x
$$


which is equal, up to a multiplicative constant, which, therefore, does not change anything in the description of the problem, to the total free energy of the two-phase model $(1.1)$,

$$
\mathcal{F}(\tilde{c})=\int_{\Omega}\left[\frac{1}{n}\left(c^{2}(1-c)^{2}\right)+\frac{\varepsilon^{2}}{2}|\nabla c|^{2}\right] d x+\frac{\lambda_{0}}{2} \int_{\Omega \backslash D}(c-h)^{2} d x,
$$

so that the model satisfies (i).

Furthermore, (ii) says that our particular $\tilde{c}$ has to be solution to (2.8)-(2.9), which is equivalent to

$$
\frac{\partial F}{\partial c_{k}}-\left.\frac{1}{n} \sum_{l=1}^{n} \frac{\partial F}{\partial c_{l}}\right|_{c_{k}=0, c_{j}=1-c_{i}}=0
$$

Moreover, we have

$$
\begin{aligned}
& \frac{\partial F}{\partial c_{k}}-\left.\frac{1}{n} \sum_{l=1}^{n} \frac{\partial F}{\partial c_{l}}\right|_{c_{k}=0, c_{i}=c, c_{j}=1-c_{i}} \\
& =-\frac{2}{n^{2}}\left[c(1-c)^{2}-c^{2}(1-c)+(1-c) c^{2}-(1-c)^{2} c\right] \\
& =0,
\end{aligned}
$$

which yields that the model satisfies (6.4) and then (ii).

\section{$7 \quad$ Numerical simulations}

As far as the numerical simulations are concerned, we rewrite the problem in the form

$$
\begin{gathered}
\frac{\partial c_{i}}{\partial t}+\Delta \mu_{i}+\lambda_{0} \chi_{\Omega \backslash D}(x)\left(c_{i}-h_{i}\right)=0, \quad i=1, \ldots, n, \\
\mu_{i}=\epsilon^{2} \Delta c_{i}-f_{i}(c), \quad i=1, \ldots, n, \\
\frac{\partial c_{i}}{\partial \nu}=\frac{\partial \mu_{i}}{\partial \nu}=0 \text { on } \Gamma, \quad i=1, \ldots, n \\
c_{\mid t=0}=c_{0}
\end{gathered}
$$

where $f_{i}(c)=\frac{2}{n}\left[c_{i}\left(1-c_{i}\right)^{2}-c_{i}^{2}\left(1-c_{i}\right)\right]-\frac{1}{n} \sum_{i=1}^{n} \frac{\partial F(c)}{\partial c_{i}}, i=1, \ldots, n$. Problem (7.1)-(7.4) has the advantage of splitting the fourth-order (in space) equation into a system of two second-order ones (see $[25,35,36]$ ). Consequently, we use a P1-finite element for the space discretization, together with a semi-implicit Euler time discretization (i.e., implicit for the linear terms and explicit for the nonlinear ones). The numerical simulations are performed with the software Freefem ++ (see [33]).

In the numerical results presented below, $\Omega$ is a $(0,0.5) \times(0,0.5)$-square. The triangulation is obtained by dividing $\Omega$ into $100 \times 100$ rectangles and by dividing each rectangle along the same diagonal.

In order to obtain the final inpainting results, we use a dynamic one step algorithm with threshold involving the diffuse interface thickness $\varepsilon$ which allows us to connect regions across large inpainting domains (see [15]). Here, the thresholding consists in replacing $\max _{i=1, \ldots, n} c_{i}$ by 1 and $c_{j} \neq \max _{i=1, \ldots, n} c_{i}$ by 0 for all $j$ and at every point $(x, y) \in \Omega$ (more generally, at every point $(x, y, z) \in \Omega$ ); this means that we replace the dominant phase (color) by 1 at every point of $\Omega$ and the other phases (colors) by 0 to obtain the final inpainting result. 


\subsection{Three colors inpainting}

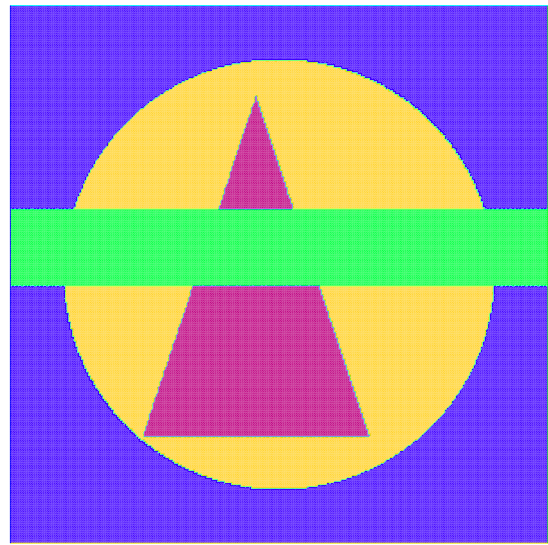

(a)

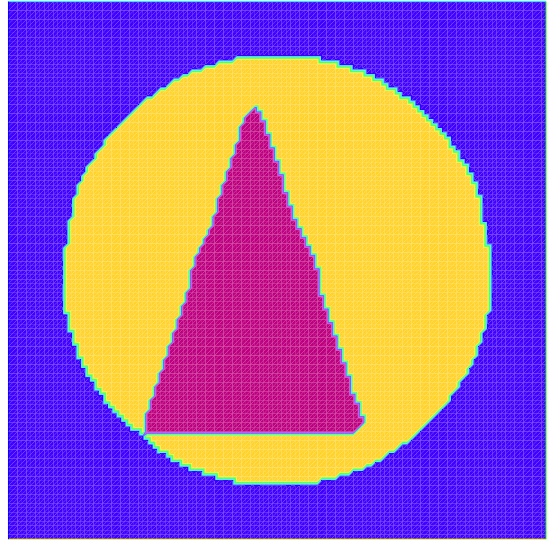

(b)

Figure 1: (a) Inpainting region in green, random initial datum between 0 and 1 in inpainting region, $\varepsilon=0.03$. (b) Solution at $t=0.006$; replacing the dominant color by 1 and the other colors by 0 .

The green region in Figure 1(a) corresponds to the inpainting region. We run the modified Cahn-Hilliard system $(n=3)$ with $\varepsilon=0.03, \Delta t=0.001$, and $\lambda_{0}=500000$. We are close to a steady state at $t=0.006$ and we replace the dominant color by 1 and the other colors by 0 to obtain the final inpainting in Figure 1(b).

\subsection{Nine colors inpainting}

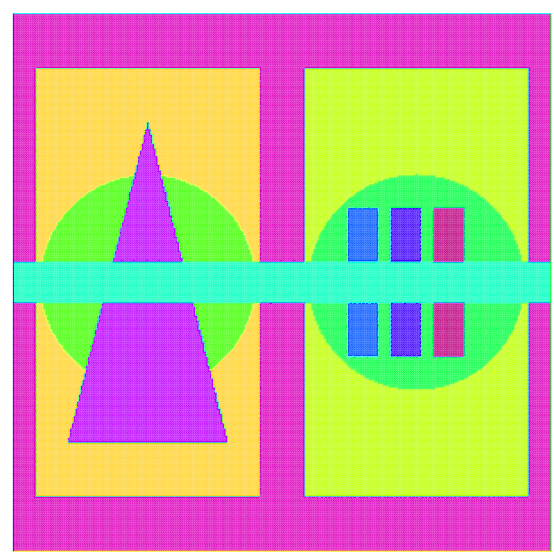

(a)

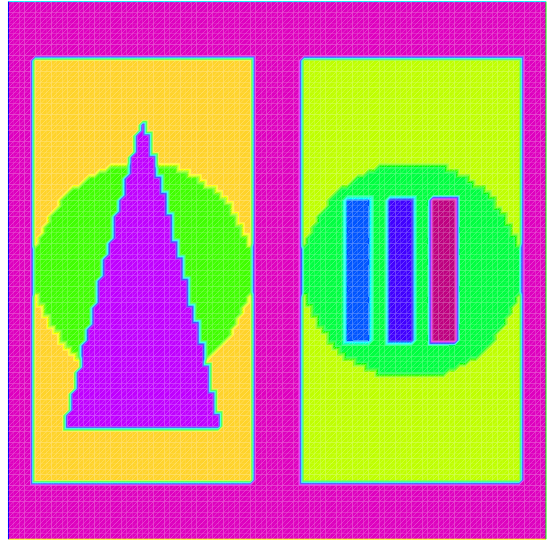

(b)

Figure 2: (a) Inpainting region in light blue, random initial datum between 0 and 1 in inpainting region, $\varepsilon=0.03$. (b) Solution at $t=0.2$; replacing the dominant color by 1 and the other colors by 0 .

In Figure 2(a), the light blue region corresponds to the inpainting region. We run the modified Cahn-Hilliard system $(n=9)$ with $\varepsilon=0.03, \Delta t=0.05$, and $\lambda_{0}=900000$. 
We are close to a steady state at $t=0.2$ and we replace the dominant color by 1 and the other colors by 0 to obtain the final inpainting in Figure 2(b).

\subsection{Text inpainting}

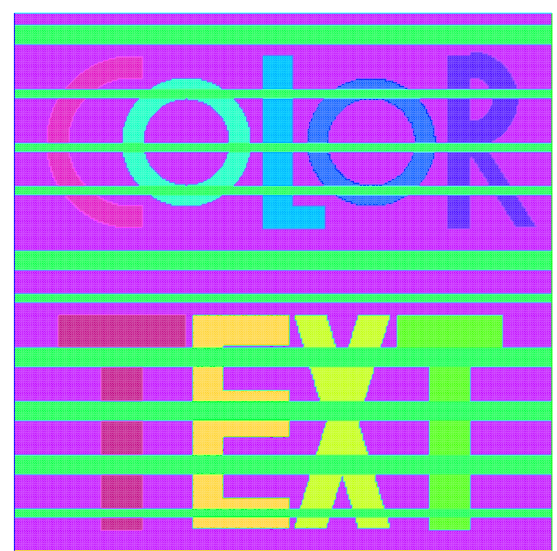

(a)

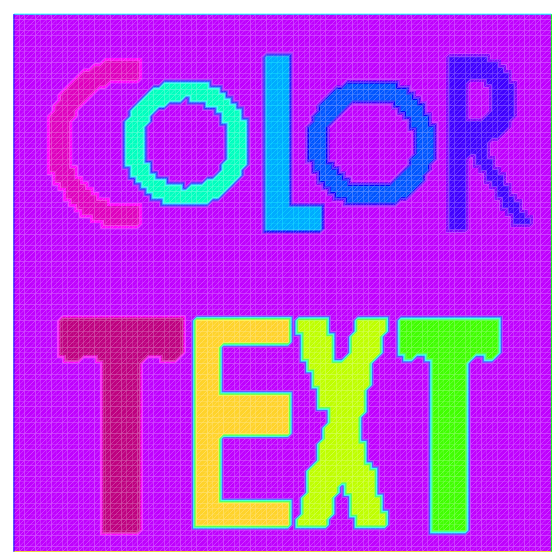

(b)

Figure 3: (a) Inpainting region in green (horizontal bars), random initial datum between 0 and 1 in inpainting region, $\varepsilon=0.008$. (b) Solution at $t=0.04$; replacing the dominant color by 1 and the other colors by 0 .

The light green region (horizontal bars) in Figure 3(a) corresponds to the inpainting region. We run the modified Cahn-Hilliard system $(n=10)$ with $\varepsilon=0.008, \lambda_{0}=$ 3000000 , and $\Delta t=0.01$. We are close to a steady state at $t=0.04$ and we replace the dominant color by 1 and the other colors by 0 to obtain the final inpainting in Figure $3(b)$.

\subsection{Consistency with the two-phase model example}

In Figure 4, we take the same example of nine colors inpainting as in Figure 2, but, now, we take $h_{i}=c_{i}(0)=0, i=1, \ldots, 7$. We run again the modified Cahn-Hilliard system $(n=9)$ with the same parameters as in Figure 2. We are close to a steady state at $t=0.2$ and we replace the dominant color by 1 and the other colors by 0 to obtain the final inpainting in Figure 4(b). Note that $\left|c_{i}(t)\right|<10^{-5}, \forall t \geq 0, i=1, \ldots, 7$.

\section{References}

[1] P.W. Bates and P.C. Fife, The dynamics of nucleation for the Cahn-Hilliard equation, SIAM J. Appl. Math. 53 (1993), 990-1008.

[2] M. Bertalmio, A. Bertozzi, and G. Sapiro, Navier-Stokes, fluid dynamics, and image and video inpainting, in Proceedings of the IEEE Computer Vision and Pattern Recognition, Vol. 1, 335-362, 2001.

[3] M. Bertalmio, G. Sapiro, V. Casselles, and C. Ballester, Image inpainting, in Siggraph 2000, Computer Graphics Proceedings, K. Akeley, ed., ACM Press/Addison-Wesley, New York, 417-424, 2000. 


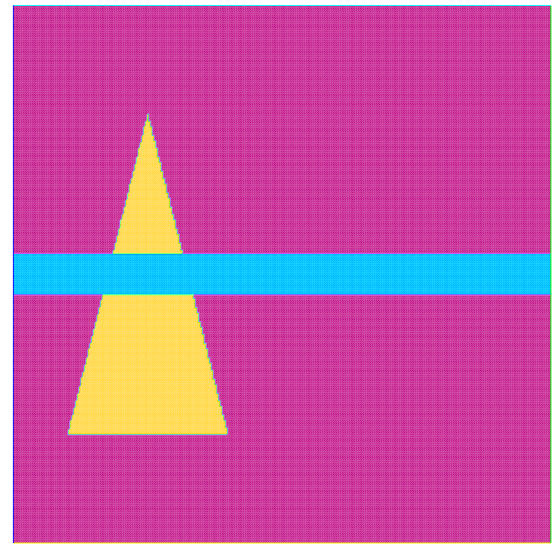

(a)

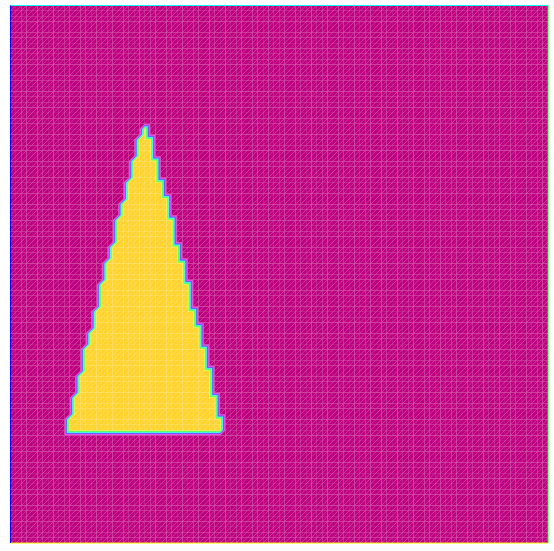

(b)

Figure 4: (a) Inpainting region in light blue, random initial datum between 0 and 1 in inpainting region, $\varepsilon=0.03$. (b) Solution at $t=0.2$; replacing the dominant color by 1 and the other colors by 0 .

[4] A. Bertozzi, S. Esedoglu, and A. Gillette, Analysis of a two-scale Cahn-Hilliard model for binary image inpainting, Multiscale Model. Simul. 6 (2007), 913-936.

[5] J. Bosch, D. Kay, M. Stoll, and A.J. Wathen, Fast solvers for Cahn-Hilliard inpainting, SIAM J. Imag. Sci. 7 (2013), 67-97.

[6] J. Bosch and M. Stoll, A fractional inpainting model based on the vector-valued Cahn-Hilliard equation, preprint.

[7] F. Boyer and C. Lapuerta, Study of a three component Cahn-Hilliard flow model, M2AN, Mod. Math. Anal. Num. 40 (2006), 653-687.

[8] C. Braverman, Photoshop retouching handbook, IDG Books Worldwide, 1998.

[9] M. Burger, L. He, and C. Schönlieb, Cahn-Hilliard inpainting and a generalization for grayvalue images, SIAM J. Imag. Sci. 3 (2009), 1129-1167.

[10] T.F. Chan, S.H. Kang, and J. Shen, Euler's elastica and cuvature-based inpainting, SIAM J. Appl. Math. 63 (2002), 564-592.

[11] T.F. Chan and J. Shen, Variationnal restoration of nonflat image features: Models and algorithms, SIAM J. Appl. Math. 61 (2001), 1338-1361.

[12] T.F. Chan and J. Shen, Mathematical models of local nontexture inpaintings, SIAM J. Appl. Math. 62 (2002), 1019-1043.

[13] T.F. Chan and J. Shen, Variationnal image inpainting, Commun. Pure Appl. Math. 58 (2005), 579-619.

[14] T.F. Chan, J. Shen, and H.M. Zhou, Total variation wavelet inpainting, J. Math. Imag. Vis. 25 (2006), 107-125.

[15] L. Cherfils, H. Fakih, and A. Miranville, Finite-dimensional attractors for the Bertozzi-Esedoglu-Gillette-Cahn-Hilliard equation in image inpainting, Inv. Prob. Imag. 9 (2015), 105-125. 
[16] L. Cherfils, H. Fakih, and A. Miranville, On the Bertozzi-Esedoglu-GilletteCahn-Hilliard equation with logarithmic nonlinear terms, SIAM J. Imag. Sci., to appear.

[17] L. Cherfils, A. Miranville, and S. Zelik, On a generalized Cahn-Hilliard equation with biological applications, Discrete Cont. Dyn. Systems B 19 (2014), 2013-2026.

[18] L. Cherfils, M. Petcu, and M. Pierre, A numerical analysis of the Cahn-Hilliard equation with dynamic boundary conditions, Discrete Cont. Dyn. Systems 27 (2010), 1511-1533.

[19] M. Conti, S. Gatti, and A. Miranville, Multi-component Cahn-Hilliard systems with dynamic boundary conditions, Nonlinear Anal. Series B: Real World Applications, to appear.

[20] J.A. Dobrosotskaya and A. Bertozzi, A Wavelet-Laplace variational technique for image deconvolution and inpainting, Trans. Imag. Proc. 17 (2008), 657-663.

[21] I.C. Dolcetta, S.F. Vita, and R. March, Area-preserving curve-shortening flows: From phase separation to image processing, Interfaces Free Bound. 4 (2002), 325343.

[22] A. Eden, C. Foias, B. Nicolaenko, and R. Temam, Expenential Attractors for Dissipative Evolution Equations, Research in Applied Mathematics, Vol. 37, JohnWiley, New York, 1994.

[23] M. Efendiev, A. Miranville, and S. Zelik, Exponential attractors for a nonlinear reaction-diffusion system in $\mathbb{R}^{3}$, C.R. Acad. Sci. Paris Série I Math. 330 (2000), $713-718$

[24] M. Efendiev, A. Miranville, and S. Zelik, Exponential attractors for a singularly perturbed Cahn-Hilliard system, Math. Nach. 272 (2004), 11-31.

[25] C.M. Elliott, D.A. French, and F.A. Milner, A second order splitting method for the Cahn-Hilliard equation, Numer. Math. 54 (1989), 575-590.

[26] C.M. Elliott, The Cahn-Hilliard model for the kinetics of phase separation, in Mathematical models for phase change problems, Birkhäuser Basel, 35-73, 1989.

[27] C. M. Elliott and S. Luckhaus, A generalised diffusion equation for phase separation of a multicomponent mixture with interfacial free energy, SFB256 University of Bonn, Preprint 195, 1991.

[28] G. Emile-Male, The restorer's handbook of easel painting, Van Nostrand Reinhold.

[29] S. Esedoglu and J. Shen, Digital inpainting based on the Mumford-Shah-Euler image model, Eur. J. Appl. Math. 13 (2002), 353-370.

[30] J.D. Eyre, Systems of Cahn-Hilliard equations, SIAM J. Appl. Math. 53 (1993), 1686-1712.

[31] J.D. Eyre, An unconditionally stable one-step scheme for gradient systems, Technical report, Department of Mathematics, University of Utah, Salt Lake City, Utah, USA, 1997. 
[32] J.D. Eyre, Unconditionally gradient stable time marching the Cahn-Hilliard equation, Mater. Res. Soc. Proceedings, Vol. 529, J.W. Bullard, L.Q. Chen, R.K. Kalia, and A.M. Stoneham, eds., Cambridge University Press, 39-46, 1998.

[33] FreeFem ++ is freely avalaible at http://www.freefem.org/ff ++ .

[34] H. Garcke, B. Nestler, and B. Stoth, On anisotropic order parameter models for multi-phase systems and their sharp interface limits, Phys. D 115 (1998), 87-108.

[35] M. Grasselli and M. Pierre, A splitting method for the Cahn-Hilliard equation with inertial term, Math. Models Methods Appl. Sci. 20 (2010), 1-28.

[36] S. Injrou and M. Pierre, Stable discretizations of the Cahn-Hilliard-Gurtin equations, Discrete Cont. Dyn. Systems 22 (2008), 1065-1080.

[37] D. King, The Commissar vanishes, Henry Holt and Company, 1997.

[38] A.C. Kokaram, Motion Picture Restoration: Digital Algorithms for Artefact Suppression in Degraded Motion Picture Film and Video, Springer Verlag, 1998.

[39] D. Li and C. Zhong, Global attractor for the Cahn-Hilliard system with fast growing nonlinearity, J. Diff. Eqns. 149 (1998), 191-210.

[40] S. Masnou and J.M. Morel, Level lines based disocclusion, in Proceedings of the 5th IEEE International Conference on Image Processing, Vol. 3, 259-263, 1998.

[41] A. Miranville and S. Zelik, Attractors for dissipative partial differential equations in bounded and unbounded domains, in Handbook of Differential Equations, Evolutionary Partial Differential Equations, Vol. 4, C.M. Dafermos and M. Pokorny, eds., Elsevier, Amsterdam, 103-200, 2008.

[42] A. Novick-Cohen and L.A. Segel, Nonlinear aspects of the Cahn-Hilliard equation, Phys. D 10 (1984), 277-298.

[43] L. Rudin, S. Osher, and E. Fatemi, Nonlinear total variation based noise removal algorithms, Phys. D 60 (1992), 259-268.

[44] L. Rudin and S. Osher, Total variation based image restoration with free local constraints, in Proceedings of the 1st IEEE ICIP, Vol 1, 259-268, 1994.

[45] J.E. Taylor and J.W. Cahn, Linking anisotropic sharp and diffuse surface motion laws via gradient flows, J. Statist. Phys. 77 (1994), 183-197.

[46] R. Temam, Infinite-Dimensional Dynamical Systems in Mechanics and Physics, 2nd ed., Springer-Verlag, New York, 1997.

[47] A. Tsai, J.A. Yezzi, and A.S. Willsky, Curve evolution implementation of the Mumford-Shah functional for image segmentation, denoising, interpolation, and magnification, Trans. Imag. Proc. 10 (2001), 1169-1186. 